\title{
SULPHATED ACID MUCOPOLYSACCHARIDE METABOLISM IN THE RABBIT INTERVERTEBRAL DISC
}

\author{
William A. Souter, Edinburgh, Scotland \\ and \\ Thomas K. F. Taylor, Sydney, Australia \\ From the Department of Orthopaedics, University of Washington School of Medicine, \\ Seattle, Washington, United States of America
}

It has been said that approximately one-third of all non-traumatic orthopaedic out-patient hospital referrals are patients with disorders of the spine (Adams 1967). In view of the frequency of degenerative disc disease among these cases it is somewhat surprising that relatively little basic research into disc metabolism and pathology at a cellular level has been reported, although it is well established on biochemical grounds that prolapse of a lumbar intervertebral disc is associated with an alteration in the composition of the extracellular matrix and degradation of the protein polysaccharide complexes in the tissue (Davidson and Woodhall 1959; Mitchell, Hendry and Billewicz 1961; Taylor and Little 1965). The physical properties of a connective tissue depend upon the integrity of the components of the extracellular matrix, primarily the collagenous fraction and the protein-polysaccharide complexes, and in the intervertebral disc the latter impart resilience to the intact structure. These complexes, in turn, can be regarded as an expression of cell function. The study of acid mucopolysaccharide synthesis at the cellular level in the intervertebral disc of a suitable experimental animal has not been reported, to our knowledge, apart from the investigation carried out by Hansen and Ullberg (1960), who employed contact autoradiography to investigate the uptake of $S^{35}$ in the intervertebral discs of suckling piglets after injection of $\mathrm{S}^{35}$ sulphate. Since microautoradiography provides more precise information, this method was chosen to study the sequence of events after the administration of radioactive sulphate to immature rabbits.

\section{MATERIALS AND METHODS}

Fourteen fully weaned four-week-old New Zealand white rabbits were injected intraperitoneally with $\mathrm{S}^{35}$ labelled sodium sulphate (New England Nuclear, Boston, Massachusetts) 1 millicurie/kilogram of body weight. The animals were killed with Dibutal at the following intervals: fifteen minutes; one, two, four, eight and twenty-four hours (two animals); four and eight days (two animals); twelve, sixteen, twenty-four and thirty-two days. Discs from the mid-thoracic spine, the thoraco-lumbar junction and the lower lumbar region were selected for study.

Preparation of histological sections-The discs with the adjoining vertebral epiphyses were removed immediately after death and fixed in 10 per cent formalin with cetyl pyridinium chloride as an additive (Conklin 1963). Decalcification was achieved with 5 per cent formic acid and each specimen was radiographed to ensure completion of the process. Routine paraffin embedding was used and sections were cut in the 5-7 $\mu$ range.

It is extremely difficult to produce high quality sections of the young rabbit disc because of the different consistency of the tissues present, the tough collagenous bundles of the annulus fibrosus tending to fragment and cause the knife blade to vibrate. In addition, the nucleus pulposus is a much swollen gel, and fixation artefacts with distortion due to imbibition by the nucleus and later to dehydration are frequent. The following histological stains were employed: 1) Harris's haematoxylin and eosin for general histological detail; 2) Alcian blue 8GS (Allied Chemical) at pH 1 to 2 for selective demonstration of sulphated acid mucopolysaccharides after Lev and Spicer (1964); 3) toluidine blue 
$0 \cdot 1$ per cent for metachromatic tissue components; and 4) periodic acid-Schiff reaction for neutral carbohydrates and glycoproteins (Gomori 1952, with omission of the bisulphite rinse).

The discs were sectioned in the horizontal, coronal and sagittal planes to produce a composite three-dimensional model of the intact structure.

Enzyme digestion procedures-Hydrated deparaffinised sections were subjected to the following digestion procedures for the study of acid mucopolysaccharides: 1) $\alpha$-amylase 0.1 grammes per cent (Worthington Biochemical Co., N.J.) in distilled water for three hours; 2) hyalase (Worthington Biochemical Co., N.J.) 1 milligram per millilitre of 0.85 per cent saline for twenty-four hours at 37 degrees Centigrade with a change of solution at twelve hours. These procedures were controlled by incubation of serial sections in reagents used to dissolve the enzymes.

Micro-autoradiography-Micro-autoradiographs were prepared by the dipping technique after Joftes and Warren (1955) using Eastman Kodak NTB3 emulsion. The exposure time for most of the sections was thirteen days, because it was found that with this period sections from all animals yielded satisfactory autoradiographs, and the maintenance of a constant exposure time allowed some quantitative assessment of the degree of radioactivity in the tissues of different animals. In animals showing the highest concentration of radioactivity further sections were also exposed for shorter periods, namely three and six days, to improve accuracy in the interpretation of the site of the label.

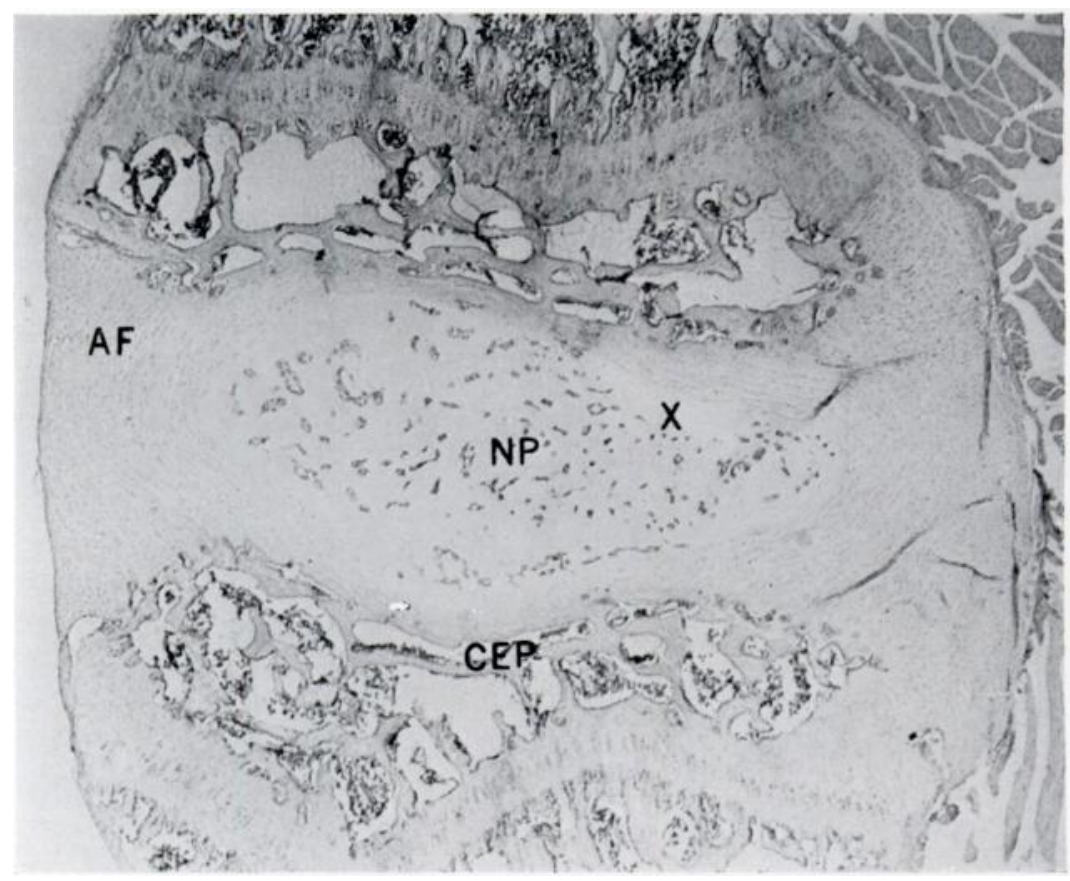

FIG. 1

Photomicrograph of the intervertebral disc of a rabbit and the adjoining vertebrae. Each vertebra is covered by a thin, plate-like epiphysis which is surmounted by the cartilage end-plate (CEP). The notochordal cells are arranged in clumps throughout the nucleus pulposus (NP) and there is an acellular band of matrix (X) between the nucleus and the annulus fibrosus (AF). (Haematoxylin and eosin, $\times 20$.)

Conversely, a few sections from each animal were exposed from four to five weeks to enhance the labelling of the micro-autoradiographs and the interpretation in sections in which the labelling obtained by the standard thirteen-day exposure was extremely light. The sections were stained either with Alcian blue after development or by periodic acid-Schiff before coating.

Contact autoradiography-Two discs removed from the thoraco-lumbar segment of the spine in each animal were immediately frozen in dry ice and acetone. Sagittal and coronal sections varying from 16 to $48 \mu$ in thickness were then cut on an International Cryostat (Model CT1). Contact autoradiographs were prepared by opposing the sections against Kodak Contrast Process Ortho film 
at -10 degrees Centigrade. The best exposure time was found to vary from three weeks in rabbits killed within twenty-four hours of injection to eight weeks for the animal killed at thirty-two days.

\section{ANATOMY OF INTERVERTEBRAL DISC IN YOUNG RABBIT}

Before proceeding to a description of the results obtained in the micro-autoradiographic study, we will describe the main anatomical characteristics of the intervertebral disc of the young rabbit.

In the four-week animal a vertebra possesses at each end a plate-like epiphysis which is covered by a cartilage end-plate (Fig. 1). The disc itself is avascular, apart from minute vessels in the extreme periphery of the annulus fibrosus (Smith and Walmsley 1951). The nucleus pulposus is highly gelatinous. The inner two-thirds of the annulus fibrosus have the microscopic features of fibrocartilage, while the outer one-third is predominantly fibrous. There are three main cell types. Firstly there are the chondrocytes of the cartilage end-plate. Secondly there are the fibrocartilaginous cells of the annulus fibrosus, which in morphological characteristics are intermediate between the chondrocyte of hyaline articular cartilage and the fibroblast. The cells from the outer one-third of the annulus fibrosus are more fibroblastic in type and lie in between the collagenous lamellae which are arranged in different directions to allow for movement. The chondrocyte-like characteristics of the cells are more pronounced in the juxta-nuclear region, where the distinct lamellar arrangement is less evident, as it is to some extent obscured by the amorphous matrix components though still readily demonstrable by polarised light examination. An occasional fibroblastic cell is seen in the nucleus pulposus. Thirdly there are the notochordal cells of the nucleus pulposus. These small cells, which have darkly staining nuclei, are irregularly arranged throughout the nucleus pulposus in clumps and, by means of branching processes, form a loose syncytium.

A striking feature of the nucleus pulposus is the acellular band of matrix which forms its periphery: further reference will be made to this area later (Fig. 1).

\section{DISTRIBUTION OF ACID MUCOPOLYSACCHARIDES IN THE RABBIT INTERVERTEBRAL DISC}

Sections stained with toluidine blue reveal intense metachromasia in the growth plate of the adjoining vertebrae, and also the cartilaginous end-plate. In the disc itself the metachromasia is less intense than in the above areas, but is still pronounced in the deeper layers of the annulus fibrosus and in the nucleus pulposus. The outer fibrous portion of the annulus fibrosus contains little metachromatic material. An almost identical staining pattern is obtained with Alcian blue and, because of the simplicity of its use, as well as the excellent contrast between the blue colour and the reduced silver grains, this stain was employed in preference to toluidine blue. The stain possesses a high degree of specificity at low $\mathrm{pH}$ for acid mucopolysaccharide. Incubation with hyalase for twenty-four hours before staining results in a drastic reduction in the Alcian blue positivity of the tissues, colour persisting only in the areas of the calcified cartilage of the growth columns, and to a lesser degree in the inner one-half to two-thirds of the annulus fibrosus.

With the periodic acid-Schiff stain a moderately positive reaction is observed in the inner two-thirds of the annulus fibrosus, while more intense staining is noted in the groups of notochordal cells in the nucleus pulposus. The latter is due in part to glycogen granules within the cells, this element being abolished by incubation in amylase. An amylase-resistant periodic acid-Schiff positive component also is present, however, and this is probably the glycoprotein of the reticular network around the notochordal cells and their processes. The most striking periodic acid-Schiff reaction is given by the cartilage end-plate and in the calcified cartilaginous cores of the epiphysial and metaphysial trabeculae. Periodic acid-Schiff positivity is unaffected by hyalase incubation.

VOL. 52 B, NO. 2, MAY 1970 


\section{RESULTS}

Micro-autoradiography*-Within fifteen minutes of intraperitoneal injection of the isotope, the label can be detected intracellularly both in the annulus fibrosus and the nucleus pulposus (Fig. 2). Annular labelling, however, is minimal, whereas some of the notochordal cells of the nucleus pulposus reveal an affinity for the isotope almost equal to that of the chondrocytes in the epiphysial growth columns. Moreover, the peripheral distribution of the active cells in the nucleus pulposus is very striking, the centrally placed cells showing little or no uptake.

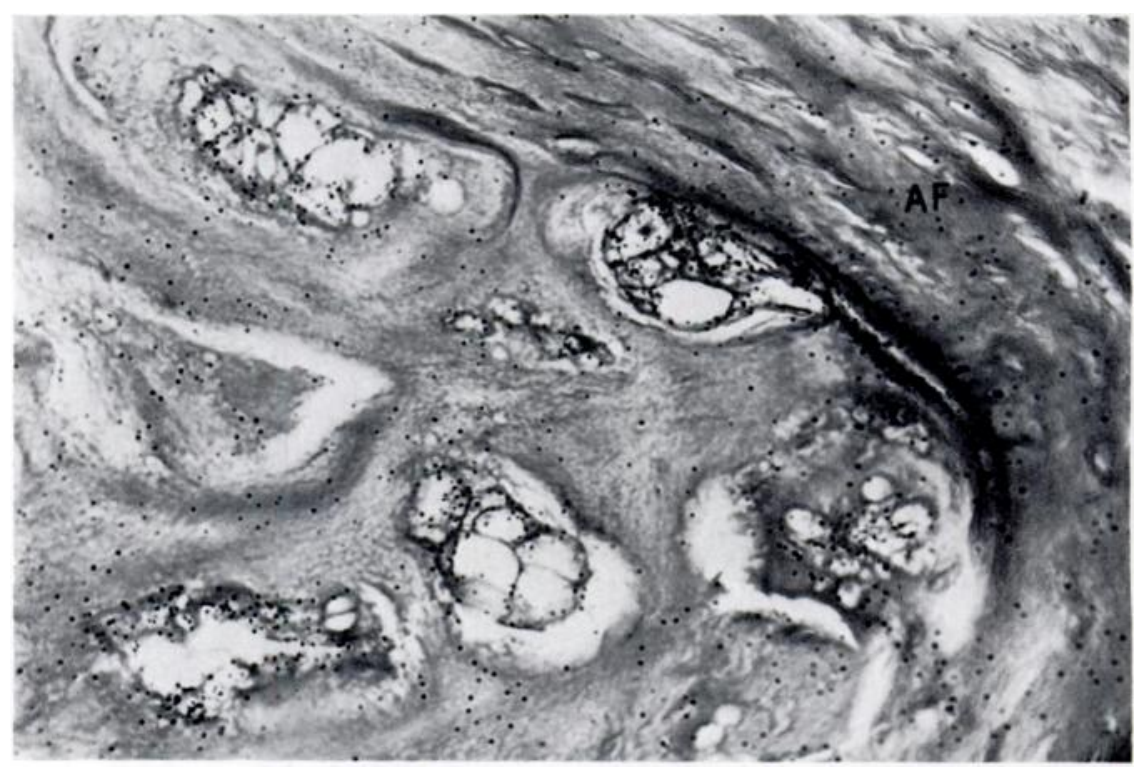

Fig. 2

A micro-autoradiograph of a section taken from the peripheral margin of the nucleus pulposus of a rabbit killed fifteen minutes after giving $\mathbf{S}^{35}$. The isotope is located intracellularly over the peripheral nuclear cells. (Alcian blue, $\times 400$.)

At one hour the above pattern of uptake of the isotope has become well established, the peripheral notochordal cells of the nucleus pulposus now showing fairly marked labelling (Fig. 3) while the inner two-thirds of the annulus fibrosus shows a punctate distribution of the label over the fibrocartilaginous cells (Fig. 4). The outer third of the annulus fibrosus shows little or no evidence of intracellular uptake of the isotope. The cells of the cartilage end-plate are also relatively inactive at this stage, with the exception of those cells towards the periphery of the epiphysis where the outer annular lamellae become anchored to the plate and of the cells around the margin of the epiphysis itself, an area we have chosen to term the peripheral epiphysial crescent.

Four hours after injection the disc presents a very striking autoradiographic picture (Fig. 5), which is further intensified at eight hours when the labelling appears to be at its maximal intensity. The intracellular position of the isotope is clearly demonstrated in the markedly punctate labelling of the annulus (Fig. 6) and in the prominent rosette of labelled groups of cells at the periphery of the nucleus (Fig. 7). At this time, in addition to the intracellular labelling of the annular lamellae, there is a heavy background of diffuse radioactivity, maximal in the outer parts of the annulus fibrosus and decreasing steadily as

\footnotetext{
*It should be stressed that in the present paper the terms "elimination", "decrease" and "persistence" as applied to the tissue radioactivity are not in any way absolute measurements but purely relative as assessed by using a thirteen-day exposure period in the preparation of the autoradiographs of the tissues taken from the fourteen different animals. Even at thirty-two days, if a sufficiently prolonged exposure is utilised, radioactivity is still detectable in all the tissues of the disc.
} 


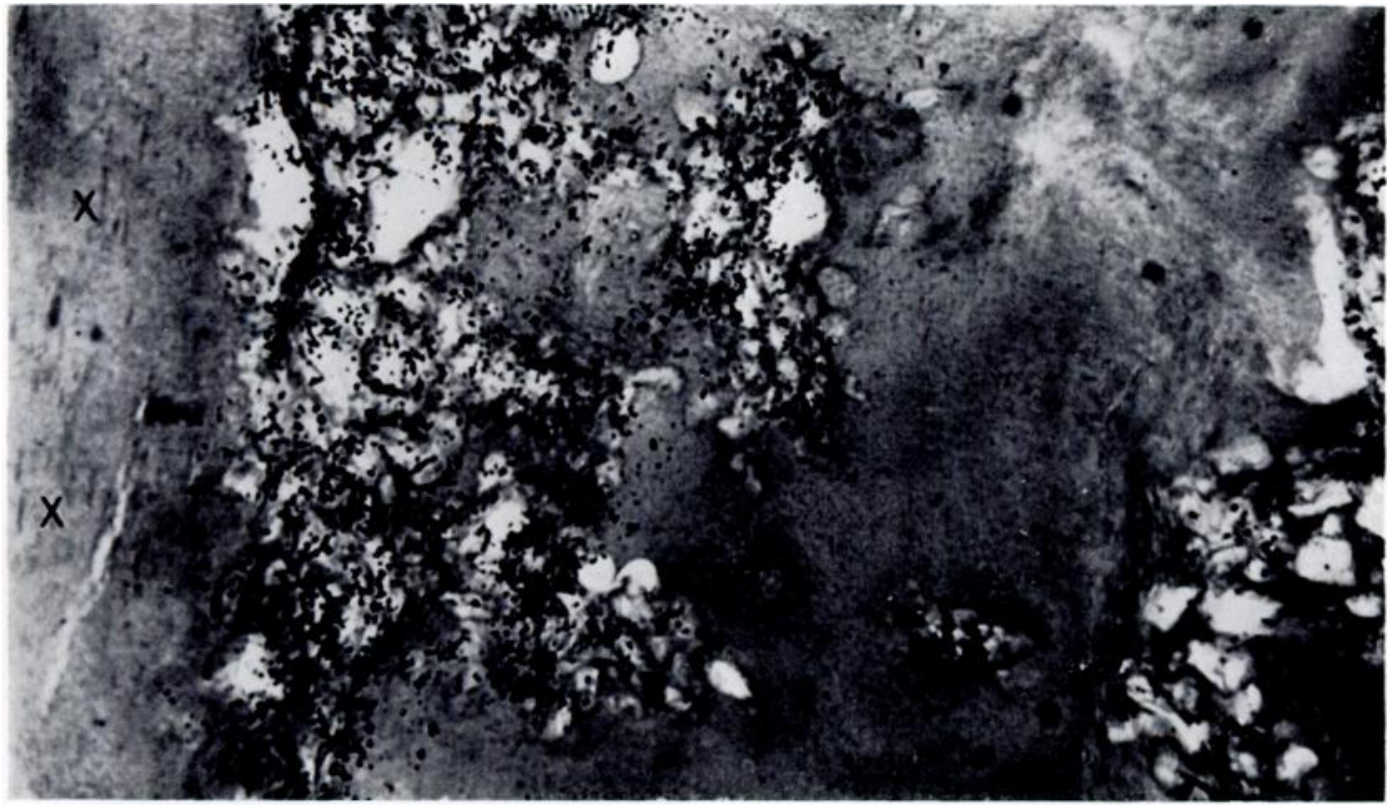

FiG. 3

Micro-autoradiograph. At one hour the cells at the margin of the nucleus show heavy intracellular labelling and those more centrally show much less. The acellular band $(X)$ is devoid of labelling. (Alcian blue, $\times 480$.)

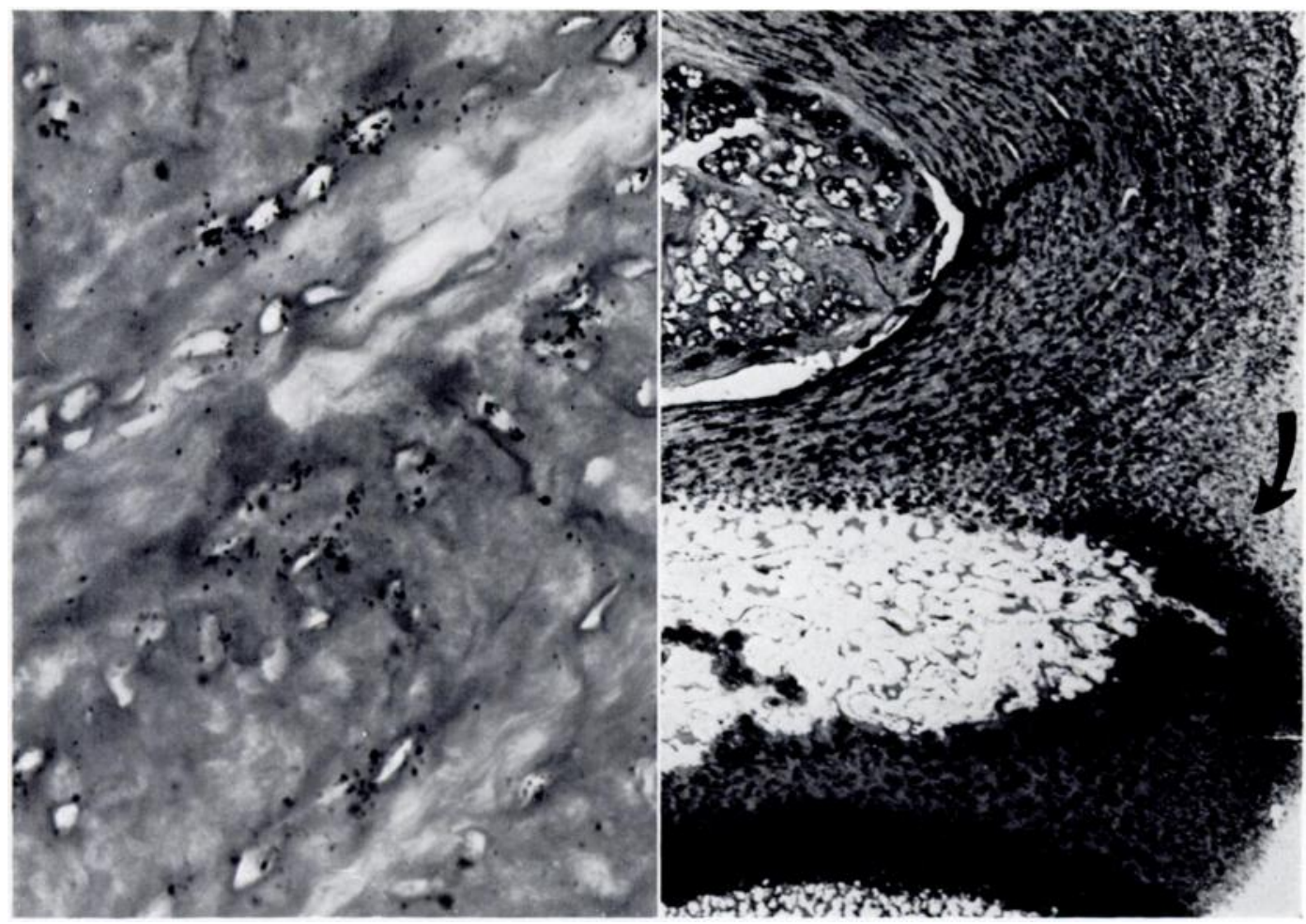

Fig. 4

FiG. 5

Figure 4-Micro-autoradiograph. At one hour after injection the fibrocartilaginous cells from the inner two-thirds of the annulus fibrosus show faint but distinct labelling. The outline of the lamellae is discernible. (Alcian blue, $\times 480$.) Figure 5-Micro-autoradiograph. At four hours after injection the peripheral notochordal cells of the nucleus pulposus are heavily labelled and the punctate distribution over the inner two-thirds of the annulus fibrosus is well demonstrated. The cells of the peripheral epiphysial crescent are also heavily labelled (arrow). The centre of the nucleus appears devoid of label. (Acian blue, $\times 45$.)

VOL. 52 B, NO. 2, MAY 1970 
the central lamellae are approached. This gradient is strongly reminiscent of the fluorescent gradient demonstrated by Brodin (1955) in his study on diffusion in the intervertebral disc using fluorochrome, and is considered to represent labelled sulphate ion in the extracellular fluid. This type of labelling can be detected within as short a time as an hour after the

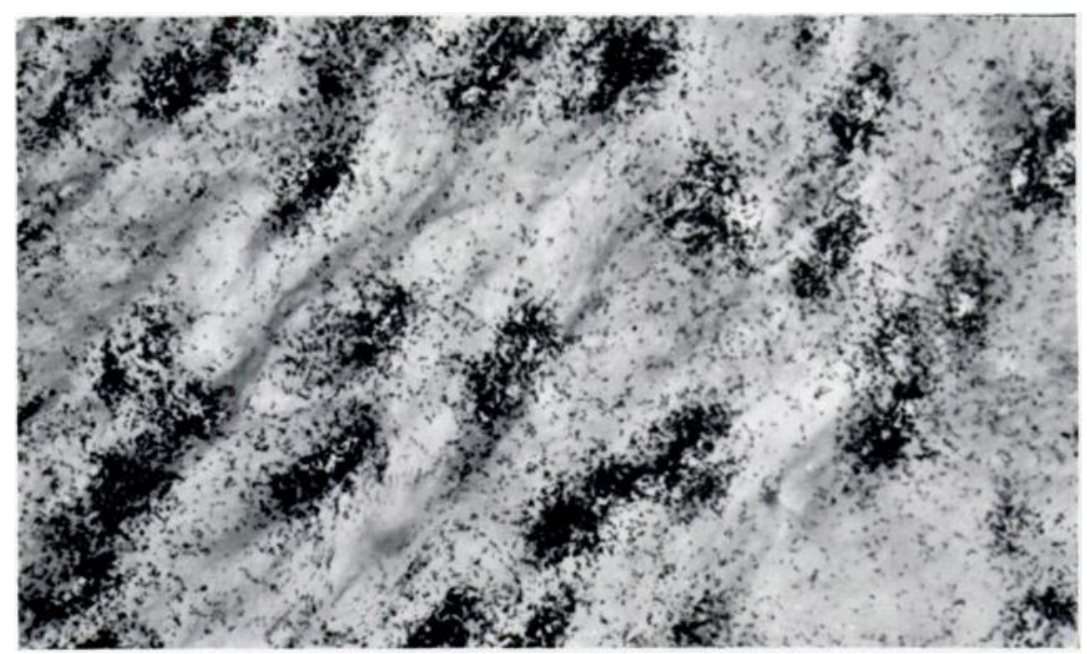

Fig. 6

Micro-autoradiograph. Intense labelling over the cells in the inner two-thirds of the annulus fibrosus is well shown. Note that not all the grains are precisely located over the cells and some are in a pericellular position. (Alcian blue, $\times 400$.)

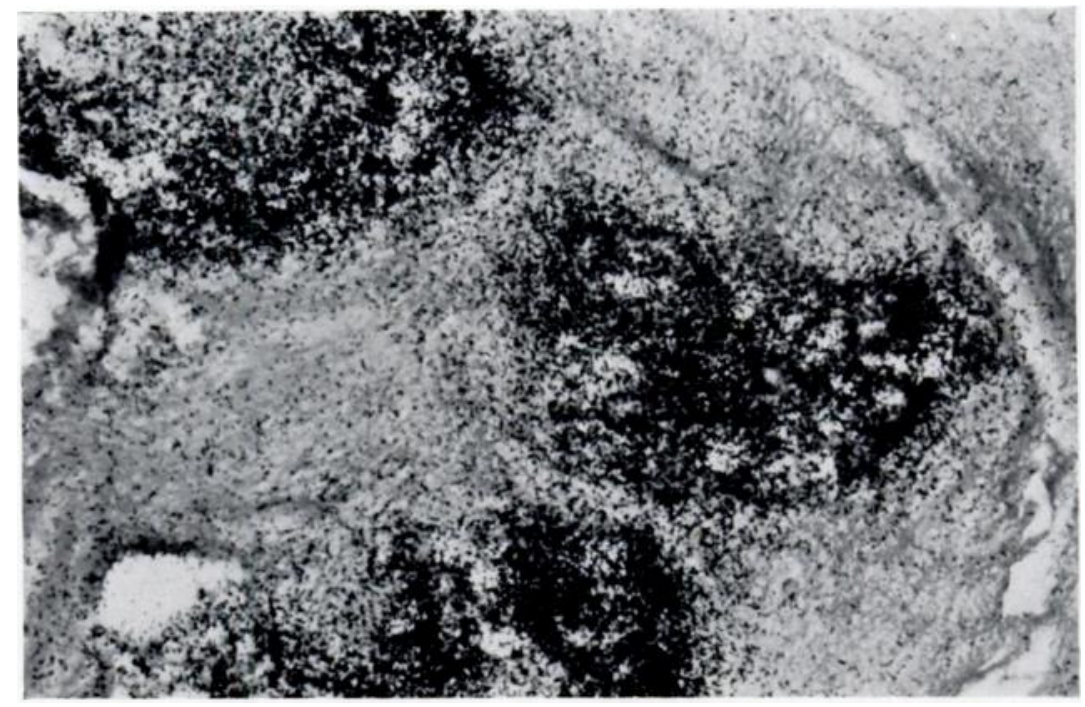

FIG. 7

Micro-autoradiograph. Eight hours after injection, heavily labelled groups of cells are shown at the nuclear periphery. (Alcian blue, $\times 400$.)

intraperitoneal injection of the isotope, provided the emulsion is exposed for four to five weeks; this background radioactivity in the disc reaches its maximum at the four to eight hour period when, incidentally, the background over the marrow of the related vertebrae is also quite high. Thereafter, there is a gradual fading of this diffuse background labelling, and it is not visible to any extent after four days from the time of injection. 
The intense intracellular labelling of the eight-hour period (Fig. 8) proves to be extremely labile to hyalase digestion, as can be seen from a comparison of Figures 8 and 9 . The

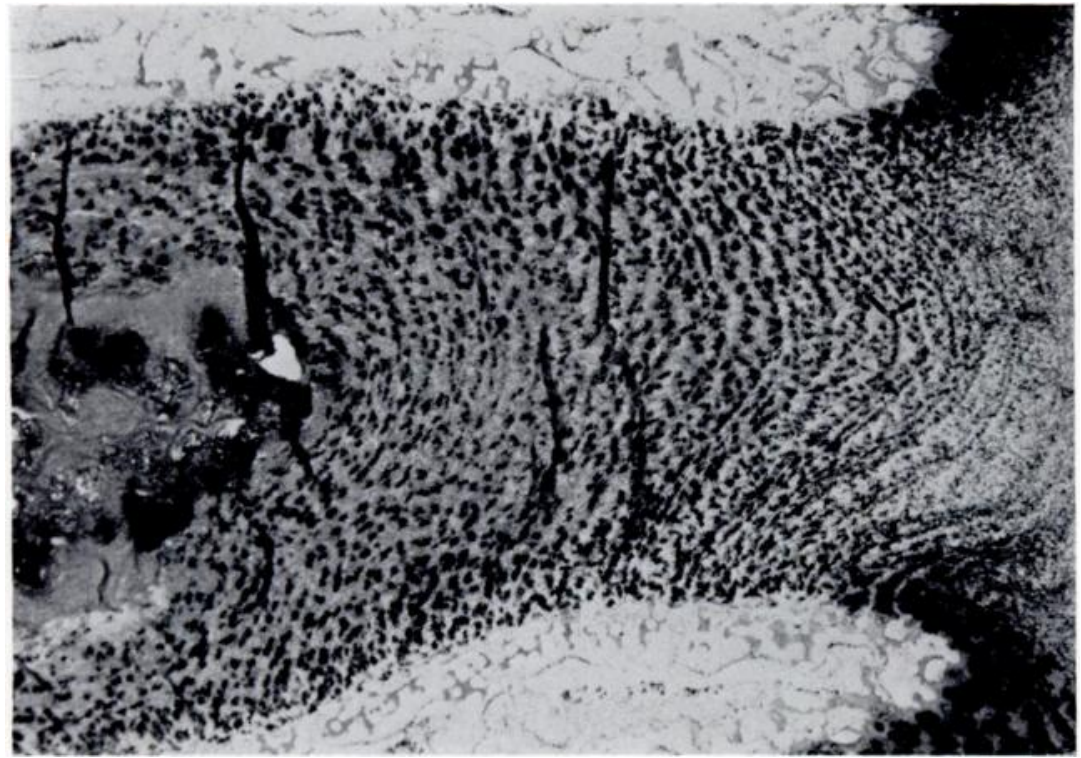

Fig. 8

Micro-autoradiograph. The intracellular location of the label at eight hours after injection is well shown both in the nucleus pulposus and the annulus fibrosus. (Alcian blue, $\times 45$.)

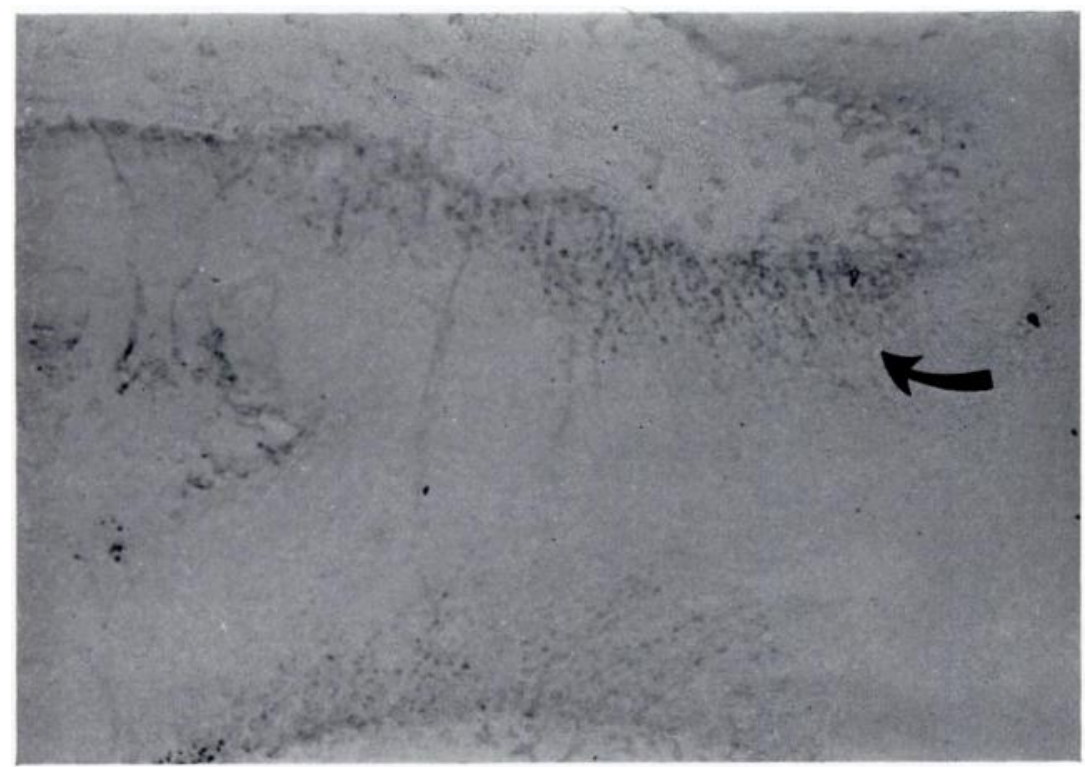

FIG. 9

Micro-autoradiograph. A section of disc comparable to that shown in Figure 8 from the eight-hour experimental period that was treated with hyalase for twenty-four hours before dipping. There is some residual activity in the peripheral nuclear cells and in the region of the peripheral epiphysial crescent (arrow). As compared with Figure 8, there is a marked reduction in alcinophilia. (Alcian blue, $\times 45$.)

radioactive source of the labelling is virtually abolished from the annular cells and persists only in very small amounts in the most heavily labelled cells in the nucleus pulposus. 
Twenty-four hours after injection the siting of the label shows definite alteration in that many of the reduced silver granules now appear to be pericellular in distribution, rather than over the cells themselves. This is clearly seen in the inner layers of the annulus fibrosus (Fig. 10) and is also apparent in the nuclear cells and in the cells of the peripheral epiphysial crescent. It seems probable that at this stage at least 50 per cent of the label is extracellular.

At the four-day interval this transposition of the label is even more complete. Now the striking features are the pericellular halos around the large clumps of peripheral cells in the

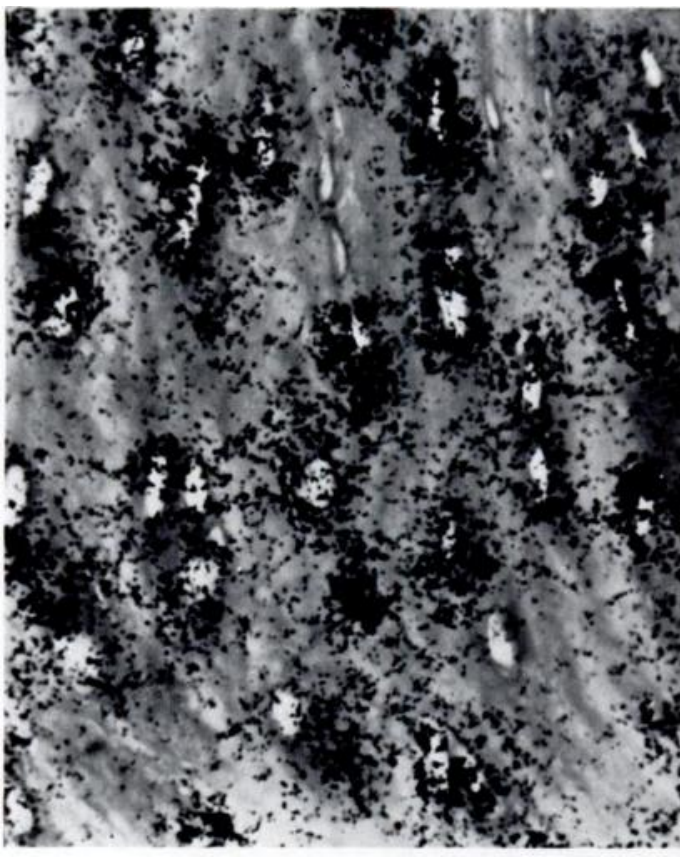

Fig. 10

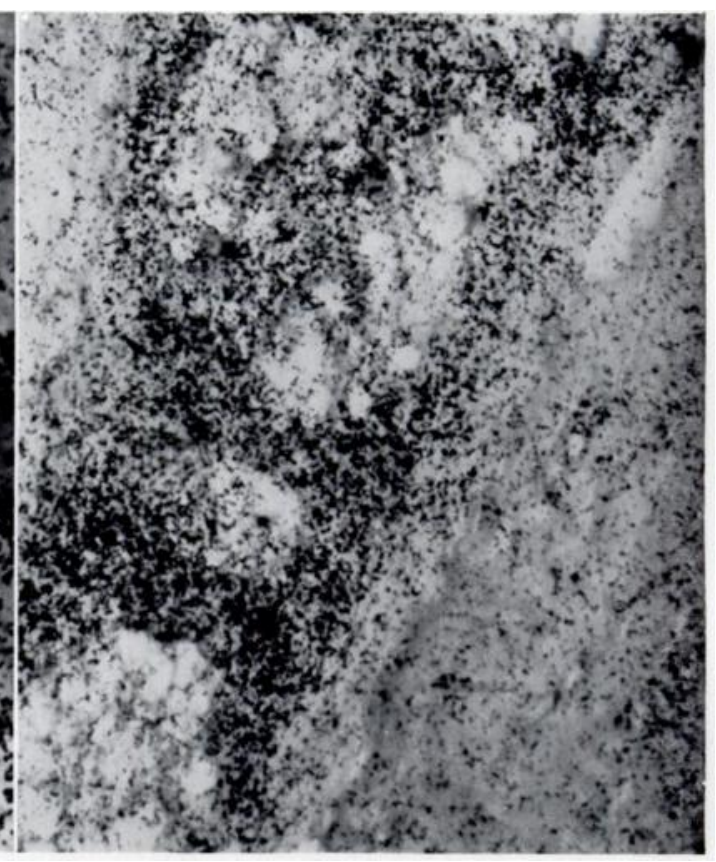

Fig. 11

Figure 10-Micro-autoradiograph. At twenty-four hours the shift of most of the label into an extracellular position is well seen in this section from the inner two-thirds of the annulus fibrosus. (Alcian blue, $\times 480$.) Figure 11-Micro-autoradiograph. At four days after injection nearly all the label in the nucleus pulposus is located extracellularly and it forms halos around the groups of notochordal cells. (Alcian blue, $\times 400$.)

nucleus pulposus (Fig. 11), while the accurate punctate labelling over the annular cells has given way to a more streaked appearance, suggestive of the outline of the annular lamellae (Fig. 12). The central area of the nucleus is still relatively clear of label. It is of interest that this also applies to the acellular band on the periphery of the nucleus, except at its central margins.

The extracellular displacement of the label is also apparent in the peripheral epiphysial crescent and in the growth columns, where the label is now clearly seen between the columns of cells and the metaphysial trabeculae, due, presumably, to the honeycomb of labelled matrix which previously ensheathed the chondrocyte columns, having been engulfed by the advancing ossification front, the labelled cartilage matrix forming the core of many of the new metaphysial trabeculae.

At the later intervals after injection of the isotope, namely, eight, twelve, sixteen, twentyfour and thirty-two days, the pericellular position of the label is lost and the grains are diffusely dispersed over the tissue, though it is true that in the annulus the labelling still preserves a somewhat streaked pattern due to the granules being concentrated along the margins of the lamellae. The diffuse spread of the labelling is seen strikingly in the nucleus pulposus (Fig. 13). As before, radioactivity is highly labile to hyalase incubation (Fig. 14). It is of interest that 


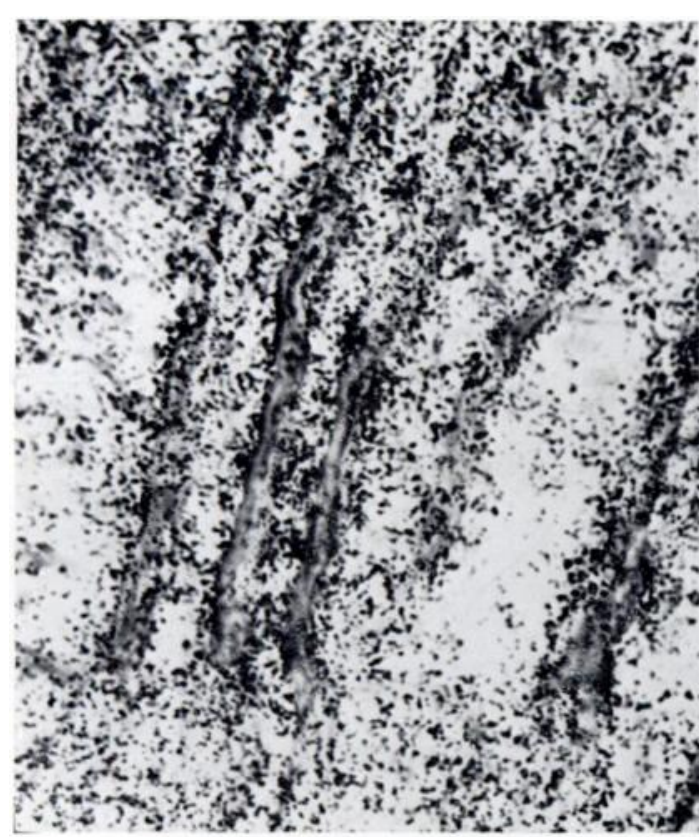

Fig. 12

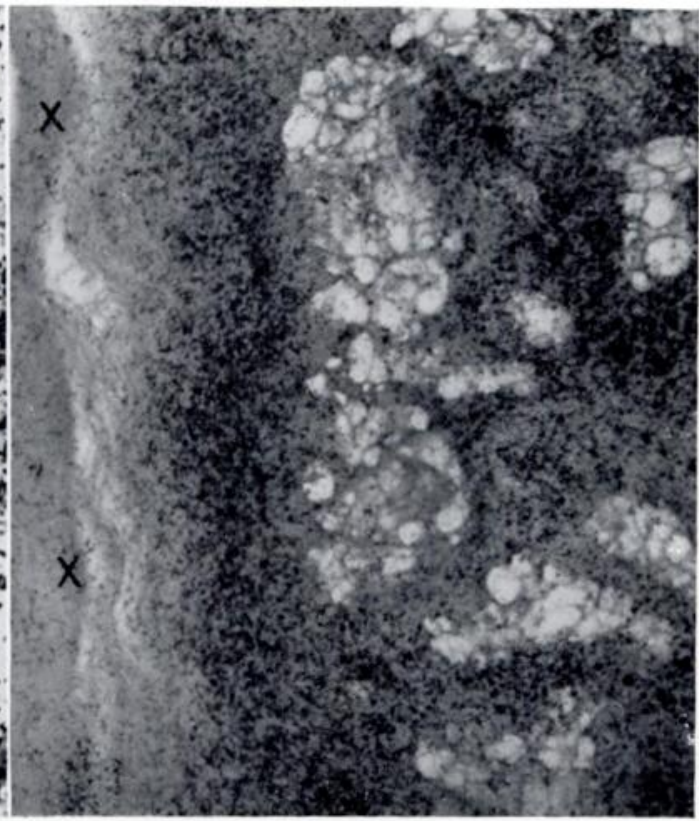

Fig. 13

Figure 12-Micro-autoradiograph. The label is diffusely spread in the annulus fibrosus, but still concentrated in a manner suggesting the lamellar arrangement. (Alcian blue, 560.) Figure 13-Micro-autoradiograph. Twelve days after injection the diffuse spread of the label, even into the central area of the nucleus pulposus, is seen, but the inner half only of the acellular band $(X)$ is labelled. (Alcian blue, $\therefore 160$.)

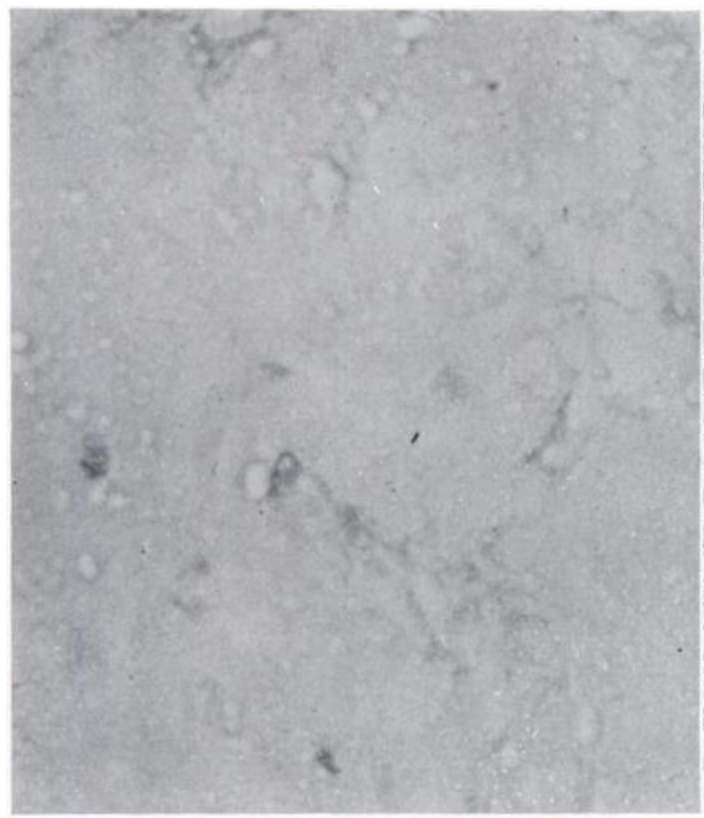

Fig. 14

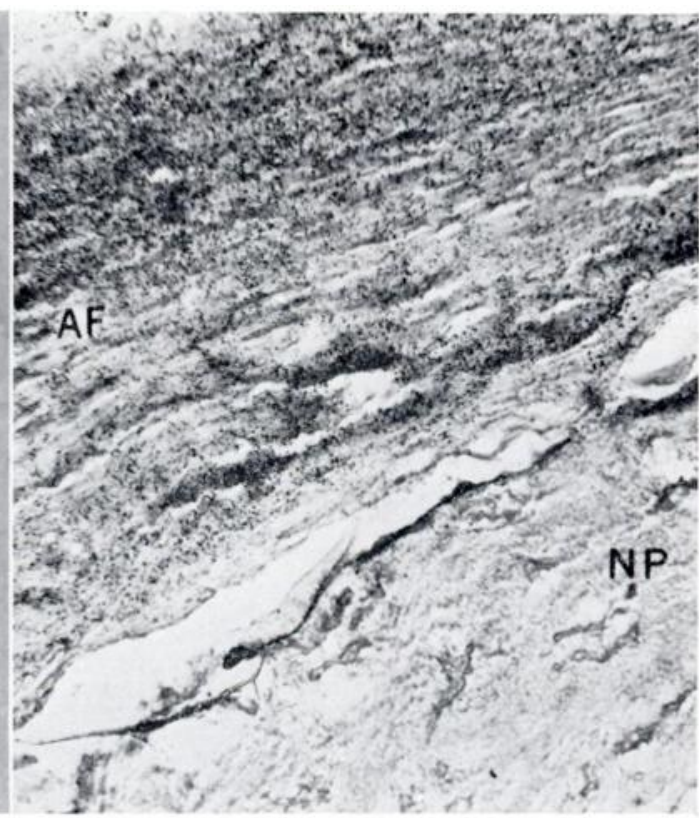

FiG. 15

Figure 14-Micro-autoradiograph. Twelve days after injection this section was treated with hyalase for twenty-four hours before dipping. There is a virtual absence of grains in the section and there is a marked reduction of alcinophilia. (Alcian blue, $: 300$.) Figure 15-Microautograph. Twenty-four days after injection diffuse and moderately heavy labelling remains in the inner two-thirds of the annulus fibrosus while the nucleus pulposus is devoid of label. (Alcian blue, $\therefore 144$.) 


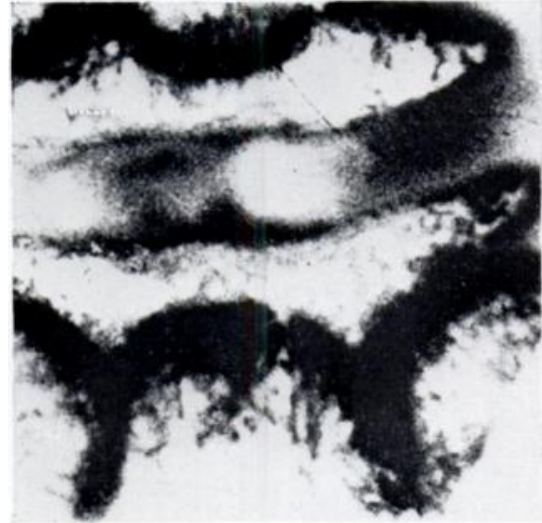

FIG. 16

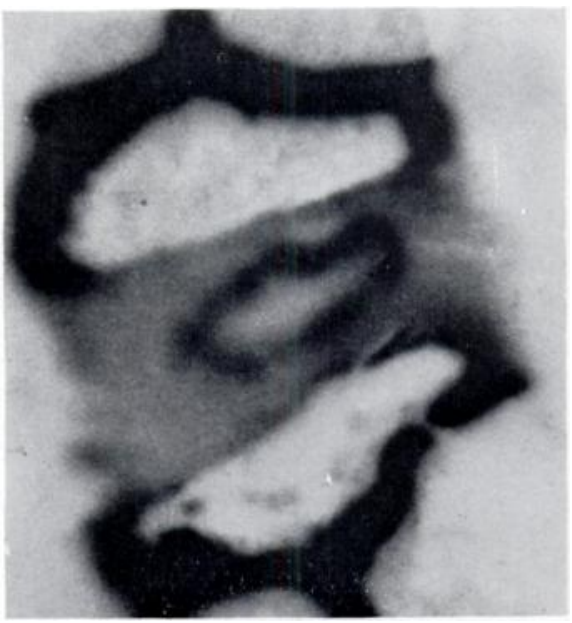

FIG. 18

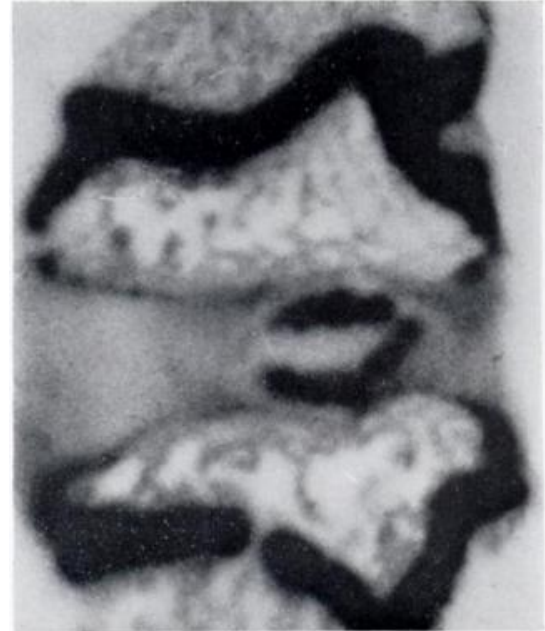

FIG. 17

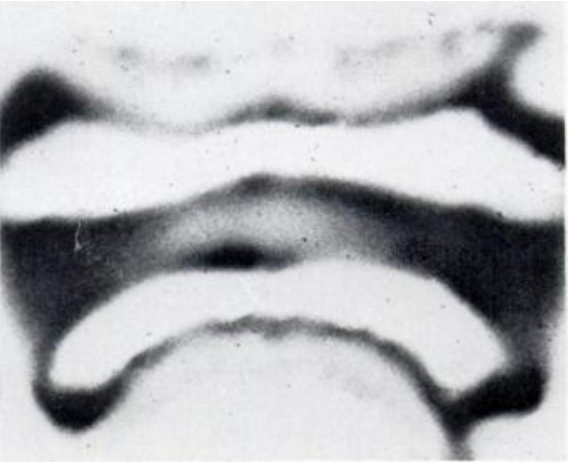

FIG. 19

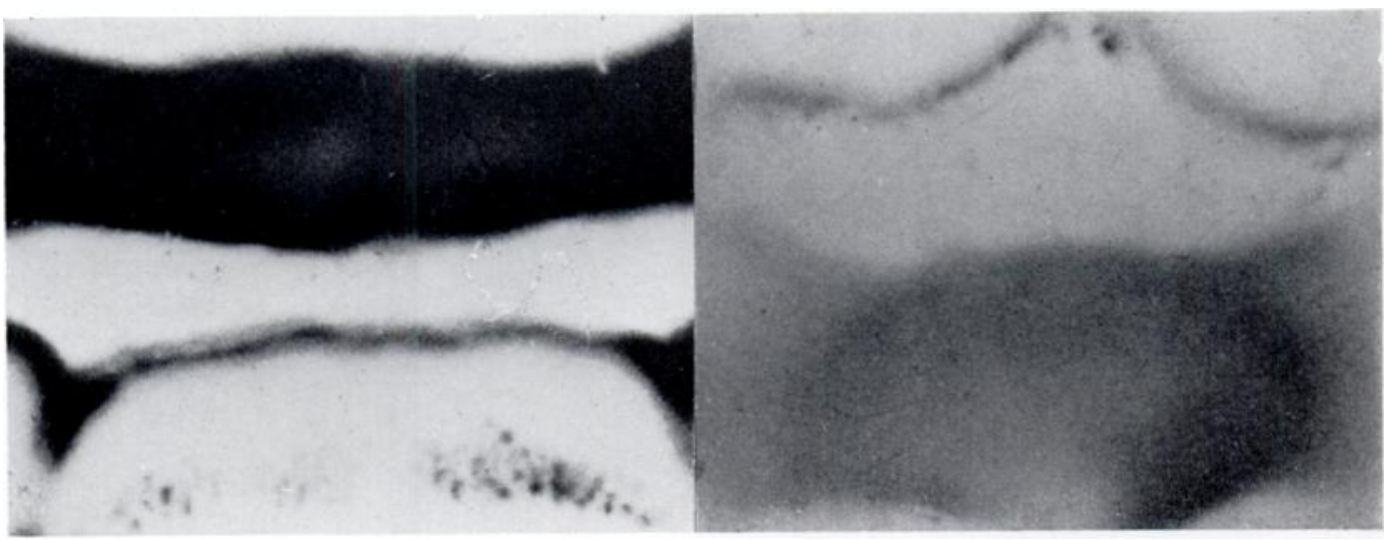

FiG. 20

FiG. 21

Contact autoradiographs of sections of intervertebral discs and the adjoining vertebral epiphyses from animals killed at: fifteen minutes (Fig. 16), eight hours (Fig. 17), twenty-four hours (Fig. 18), eight days (Fig. 19), twelve days (Fig. 20) and thirty-two days (Fig. 21) after injection of $\mathrm{S}^{35}$. See text for details. ( $\left.\therefore 10.\right)$ 
the acellular band still shows relatively little labelling except in its inner half in immediate juxtaposition to the peripheral nuclear cells.

After the twelve-day period there is a steady decrease in the intensity of radioactivity in all areas of the disc, but this decrease is by no means uniform. Whereas at sixteen days the nucleus pulposus is only very slightly labelled and shows virtually no evidence of radioactivity at twenty-four days (Fig. 15), the annular labelling proves more persistent, being still quite heavy at twenty-four days, and, though greatly reduced, is still readily apparent in most areas of the inner two-thirds of the annulus fibrosus at thirty-two days.

The cells of the peripheral epiphysial crescent exhibit a falling gradient of label intensity very similar to that of the nucleus pulposus in terms of time sequence, but it is to be remembered that here, as in the growth columns of the epiphysial plate, an interpretation of the results must make allowance for the fact that growth and ossification are apparently proceeding very actively in this area, so that what might be called a geographic displacement of the originally labelled matrix is partially responsible for the disappearance of the evidence of radioactivity from the pericellular matrix of the new generation of cells in the peripheral epiphysial crescent. Contact autoradiography-Close corroboration of the events described above was observed in the contact autoradiographs. At fifteen minutes high background activity over the tissue is evident but marked concentration of the isotope is seen in the epiphysial growth plate, the inner part of the annulus fibrosus and the periphery of the nucleus pulposus (Fig. 16).

At eight hours the contact autoradiographs closely parallel the micro-autoradiographs with intense radioactivity in the growth plate, peripheral epiphysial crescent, and less markedly in the inner part of the annulus fibrosus. Even the outline of the active peripheral groups of nuclear cells is suggested by the photographic image (Fig. 17 and compare Fig. 5). There is high background over the outer annulus fibrosus and over the adjoining vertebrae. Hyalase digestion almost abolished radioactivity from the tissue, that which remained at this time having the same distribution that is present in the micro-autoradiographs of disc tissue treated in a comparable fashion (Figs. 8 and 9).

At twenty-four hours the peripheral clumps of cells have become less discrete, again paralleling the micro-autoradiographic picture and heralding the onset of the distinct extracellular phase (Fig. 18). The apparent progressive dispersion of the radioactive label in the tissue is clearly seen (Figs. 19 and 20). The levels of radioactivity in the disc fall steadily as the intervals from the time of injection increase. But here again there is a disparity between the rate of elimination from the annulus fibrosus and the nucleus pulposus. Persistent activity in the annulus fibrosus is clearly delineated at thirty-two days (Fig. 21), and at a much lower level in the nucleus pulposus. However, absolute activity in the annulus fibrosus has diminished considerably, eight weeks of exposure being required to produce a satisfactory autoradiograph, whereas, in specimens from animals sacrificed within four days of isotope injection, two and one-half to three weeks were ample exposure times.

\section{DISCUSSION}

The principal obstacle in the path of meaningful research into the biophysiology of the human intervertebral disc is the lack of an entirely suitable experimental animal. The vertebral anatomy of man is unique and the human vertebra cannot be considered as a short long bone. Moreover, the adoption of the upright stance has imposed mechanical stresses on the intervertebral discs of man which are not found elsewhere in the animal kingdom. However, in many respects, as pointed out by Smith and Walmsley (1951), the intervertebral disc of the rabbit fulfils the purpose of an experimental model. In this animal the notochordal cells and their enveloping gelatinous matrix appear to remain relatively unaltered until two years of age although, according to the above authors, a fibrocartilaginous change supervenes later in life. Davidson and Small (1963) showed a time-dependent reversal of the keratan sulphatechondroitin sulphate ratio (determined as glucosamine-galactosamine ratios) in the nucleus

VOL. 52 B, NO. 2, MAY 1970 
pulposus of the rabbit, the ratio rising from 0.4 at one month to approximately 2.8 at twenty months. A similar, though less marked, reversal of the keratan sulphate-chondroitin sulphate ratio in the human nucleus pulposus with ageing has been demonstrated by Hallen (1958). However, the two situations are not at all comparable since in humans the notochordal elements are no longer present after the middle of the second decade, the nucleus thereafter undergoing fibrocartilaginous transformation. A study of the acid mucopolysaccharide metabolism in the annulus fibrosus in ageing, both in man and in rabbits, is indicated but, as far as we are aware, this has not yet been reported. Antonopoulos, Gardell, Szirmai and De Tyssonsk (1964), who developed a microcolumn technique for polysaccharide separation, have shown that, in the elderly rabbit, the concentration of keratan sulphate rises progressively from the periphery of the annulus to the centre of the disc, and confirmed the presence of this matrix component in the inner two-thirds of the annulus fibrosus. However, since there are such striking differences between the fibrocartilaginous cell of the annulus fibrosus and the notochordal cells of the nucleus pulposus, as well as in the physical properties of their respective matrices, there is no real reason to suppose that the keratan sulphate and its protein partner in these two adjoining segments of the disc are identical molecules. For example, it has been established that there are significant differences in the degrees of sulphation of keratan sulphates in a variety of connective tissues (Cifonelli, Saunders and Gross 1967).

The use of $\mathrm{S}^{35}$ labelled sulphate as a research tool for the study of sulphated acid mucopolysaccharide synthesis in cartilaginous tissues has gained wide acceptance following the early biochemical work of Dziewiatkowski (1949), Dziewiatkowski, Benesch and Benesch (1949) and Böstrom (1952), as well as the micro-autoradiographic studies of Dziewiatkowski (1951, 1952) and Bélanger (1954). In articular cartilage, Bélanger observed that the isotope first appeared intracellularly and then, after an interval of twenty-four to forty-eight hours, in the extracellular matrix. This biphasic siting of the label has been confirmed in studies on visceral and skeletal hyaline cartilage by several other workers (Pelc and Glücksmann 1955; Mancini, Nunez and Lusting 1956).

That the vast bulk of the isotope is indeed used intracellularly in the synthesis of chondroitin sulphate was confirmed by Dziewiatkowski (1962), who was able to show that 64 to 83 per cent of the radioactivity in cartilage slices incubated with $\mathrm{S}^{35}$ for four hours was present in chondroitin sulphate while, by collateral micro-autoradiography, it was estimated that 85 per cent of the isotope was still intracellular in position. Additional evidence of this metabolic pathway has been obtained by Thorp and Dorfman (1963), who used a suspension of chondrocytes, obtained by trypsinisation of cartilage, and found that after ten minutes' incubation in medium containing $\mathrm{S}^{35}$ the intracellular chondroitin sulphate fraction obtained by ultracentrifugation was heavily labelled with $\mathrm{S}^{35}$, whilst the extracellular labelling was slight. After incubation for one hour the intracellular labelling was unchanged but the extracellular fraction of the polysaccharide was heavily labelled. More recently it has been shown by electron microscope autoradiography that sulphation of the chondroitin sulphate molecule, or its precursor, takes place in the Golgi apparatus, the sulphated complex thereafter being extruded into the matrix via temporary stomata in the cell membrane (Godman and Lane 1964).

The distribution of the radioactive label in the polysaccharide moieties of the rabbit intervertebral disc is not shown in this study, but it is highly probable that most of it is in chondroitin sulphate, since at this age the structure contains but small amounts of keratan sulphate (Davidson and Small 1963). This is also strongly suggested by the close geographic correlation between alcinophilia and the labelling of the disc tissues as well as their parallel decrease after hyalase incubation. Further, the observed sequence of events is closely similar to that which has been recorded for hyaline articular cartilage as reviewed above.

The sojourn of the isotope in the tissues possesses an intracellular phase of approximately twenty-four hours, followed by an extracellular phase. The differing affinity of the cells for 
the sulphate ion is a very striking phenomenon, especially with regard to the peripheral cells of the nucleus pulposus. It is likely that these cells are responsible for the production of the greater part of the sulphated acid mucopolysaccharide of the nucleus pulposus. The localised pericellular halos of intense labelling seen at the four-day period, and the very diffuse matrix labelling noted in the twelve-day sections, suggest that the sulphated acid mucopolysaccharide may be secreted principally by these peripheral cells and then becomes more widely distributed throughout the whole nuclear gel. Mention has been made of the peripheral acellular band of Alcian blue positive material around the edge of the nucleus, and it appears that the formed polysaccharide molecule penetrates this band rather more slowly than it becomes distributed throughout the more central nuclear matrix. Possibly the acellular band may have diffusion properties which are different from the remainder of the nucleus.

In the early experimental time periods it was noted that the cells of the annulus fibrosus showed little or no labelling, especially when compared to the cells of the outer nucleus pulposus. There are two possible explanations. Either a longer latent period exists before the cells take up the isotope, or the isotope is quantitatively reduced to a level at which initially the presence of the isotope cannot be detected in the micro-autoradiograph. That the second explanation is the correct one is supported by the fact that after exposing the emulsion to the tissues for four or five weeks the resultant autoradiographs show quite prominent labelling of the annular cells even at fifteen minutes after injection.

The findings in the present study correlate well with those of Hansen and Ullberg (1960) in their experiments on suckling piglets which they studied up to sixteen days after the administration of the isotope. They stated that at least one day should elapse following injection of the isotope to obtain distinct autoradiographs, but in the present study this was not found to be the case. In the piglet and the rabbit the most active areas in terms of sulphate uptake are the outer rim of the nucleus pulposus and the inner two-thirds of the annulus fibrosus. The elimination of the isotope of the nucleus pulposus at twenty-four days and the persistence of the label in the annulus fibrosus at thirty-two days suggests that the metabolic turnover of polysaccharides is considerably slower in the annulus than in the nucleus pulposus.

\section{SUMMARY AND CONCLUSIONS}

1. The uptake of $\mathrm{S}^{35}$ labelled sodium sulphate has been studied autoradiographically in the intervertebral disc of the young rabbit.

2. The sojourn of the isotope in the tissues includes an intracellular phase of approximately twenty-four hours, followed by an extracellular phase.

3. The cells exhibiting by far the greatest affinity for the sulphate ion are the peripheral groups of cells of the nucleus pulposus, while the chondrocyte-like cells of the cartilaginous segment of the annulus fibrosus are also fairly active. The central cells of the nucleus and the fibroblasts of the outer one-third of the annulus have a much lower uptake.

4. By analogy with similar studies on hyaline cartilage, and on the basis of correlation between the alcinophilia of the tissues and the concentration of the label, both before and after hyalase digestion of the tissue, it is considered that in the young rabbit disc, as in articular cartilage, the sulphate is incorporated primarily into chondroitin sulphate.

5. The elimination of the isotope from the nucleus at twenty-four days and the persistence of the label in the annulus fibrosus at thirty-two days tends to suggest that the metabolic turnover of acid mucopolysaccharide is considerably slower in the annulus than in the nucleus.

The authors wish to thank Mrs S. Latimer and Mrs T. Byrd for technical assistance and also Mr M. Devlin for help with the photomicrographs. This work was supported by a Public Health Service Grant Number AM 06363-08. One of us (W. A. S.) was also in receipt of a 1967 Wellcome Travelling Fellowship.

VOL. 52 B, NO. 2, MAY 1970 


\section{REFERENCES}

Adams, J. C. (1967): Outline of Orthopaedics. Sixth edition. Edinburgh and London: E. \& S. Livingstone Ltd.

Antonopoulos, C. A., Gardell, S., Szirmai, J. A., and De Tyssonsk, E. R. (1964): Determination of Glycosaminoglycans (Mucopolysaccharides) from Tissues on the Microgram Scale. Biochimica et Biophysica Acta, 83, 1.

BÉLANGer, L. F. (1954): Autoradiographic Visualisation of the Entry and Transit of $\mathrm{S}^{35}$ in Cartilage, Bone and Dentine of Young Rats and the Effect of Hyaluronidase in Vitro. Canadian Journal of Biochemistry and Physiology, 32, 161.

Böstrom, H. (1952) On the Metabolism of the Sulphate Group of Chondroitinsulphuric Acid. Journal of Biological Chemistry, 196, 477.

Brodin, H. (1955): Paths of Nutrition in Articular Cartilage and Intervertebral Discs. Acta Orthopaedica Scandinavica, 24, 177.

Cifonelli, J. A., Saunders, A., and Gross, J. I. (1967): Keratan Sulfate Fractions from Bovine and Human Tissues. Carbohydrate Research, 3, 478.

Conklin, J. L. (1963): Staining Reactions of Mucopolysaccharides After Formalin-Containing Fixatives. Stain Technology, 38, 56.

Davidson, E. A., and Small, W. (1963): Metabolism in Vivo of Connective Tissue Mucopolysaccharides. 1. Chondroitin Sulfate $C$ and Keratosulfate of Nucleus Pulposus. Biochimica et Biophysica Acta, 69, 445.

Davidson, E. A., and Woodhall, B. (1959): Biochemical Alterations in Herniated Intervertebral Disks. Journal of Biological Chemistry, 234, 2951.

Dziewiatkowski, D. D. (1949): Rate of Excretion of Radioactive Sulfur and Its Concentration in Some Tissues of the Rat after Intraperitoneal Administration of Labeled Sodium Sulfate. Journal of Biological Chemistry, 178, 197.

DzIEwIATKowSKI, D. D. (1951): Radioautographic Visualisation of Sulfur-35 Disposition in the Articular Cartilage and Bone of Suckling Rats Following Injection of Labeled Sodium Sulfate. Journal of Experimental Medicine, 93, 451.

DzIEwIATKowskI, D. D. (1952): Radioautographic Studies of Sulfate-Sulfur $\left(\mathbf{S}^{35}\right)$ Metabolism in the Articular Cartilage and Bone of Suckling Rats. Journal of Experimental Medicine, 95, 489.

DzIEwiatkowski, D. D. (1962): Intracellular Synthesis of Chondroitin Sulfate. Journal of Cell Biology, 13, 359 .

Dziewiatkowski, D. D., Benesch, R. E., and Benesch, R. (1949): On the Possible Utilization of Sulfate Sulfur by the Suckling Rat for the Synthesis of Chondroitin Sulfate as Indicated by the Use of Radioactive Sulfur. Journal of Biological Chemistry, 178, 931.

Godman, G. C., and LaNe, N. (1964): On the Site of Sulfation in the Chondrocyte. Journal of Cell Biology, 21, 353.

Gomori, G. (1952): Microscopic Histochemistry. Chicago, Illinois: University of Chicago Press.

Hallen, A. (1958): Hexosamine and Ester Sulphate Content of the Human Nucleus Pulposus at Different Ages. Acta Chemica Scandinavica, 12, 1869.

HANSEN, H.-J., and UllberG, S. (1960): Uptake of $S^{35}$ in the Intervertebral Discs After Injection of $S^{35}$ Sulphate. An Autoradiographic Study. Acta Orthopaedica Scandinavica, 30, 84.

JofTES, D. L., and WARREN, S. (1955): Simplified Liquid Emulsion Radioautography. Journal of the Biological Photographic Association, 23, 145.

LeV, R., and SPICER, S. S. (1964): Specific Staining of Sulphate Groups with Alcian Blue at Low pH. Journal of Histochemistry and Cytochemistry, 12, 309.

Mancini, R. E., Nunez, C., and Lusting, E. S. (1956): A Radioautographic Study on the Specificity and Sequence of the Uptake of $S^{35}$ by the Mucopolysaccharide of the Embryonic and Adult Connective Tissue. Journal of Histochemistry and Cytochemistry, 4, 444.

Mitchell, P. E. G., Hendry, N. G. C., and Billewicz, W. Z. (1961): The Chemical Background of Intervertebral Disc Prolapse. Journal of Bone and Joint Surgery, 43-B, 141.

Pelc, S. R., and Glücksmann, A. (1955): Sulphate Metabolism in the Cartilage of the Trachea, Pinna and Xiphoid Process of the Adult Mouse as Indicated by Autoradiographs. Experimental Cell Research, 8, 336.

Smith, J. W., and Walmsley, R. (1951): Experimental Incision of the Intervertebral Disc. Journal of Bone and Joint Surgery, 33-B, 612.

TAYLOR, T. K. F., and LitTle, K. (1965): Intercellular Matrix of the Intervertebral Disk in Ageing and in Prolapse. Nature, 208, 384.

ThORP, F. K., and DoRfman, A. (1963): The Occurrence of Intracellular Chondroitin Sulfate. Journal of Cell Biology, 18, 13. 